\title{
Families of Riemann surfaces and Weil-Petersson geometry
}

\author{
Scott A. Wolpert
}

April 11, 2008

\section{Considerations}

The investigation of the geometry and deformation theory of Riemann surfaces is long recognized for involving a wide range of concepts and a wide range of techniques. For surfaces of negative Euler characteristic, the WeilPetersson metric provides a Hermitian structure for the space of infinitesimal variations of hyperbolic metrics. The Weil-Petersson geometry is recognized for providing important information on the geometry of the Teichmüller space and the moduli space of Riemann surfaces. In less than a decade there have been a collection of breakthrough works on Weil-Petersson geometry. The works are based on a range of approaches and so involve matters of interest and further study for a broad audience.

The plan for the lecture topics is presented in Section 5. The approach will include the fundamentals and central underlying arguments for three areas: explorations of the $C A T(0)$ geometry, applications of volume integrals and applications of curvature. Supporting goals for the approach are to present results that are based on standardized techniques and to present results that lead directly into research questions.

\section{Weil-Petersson background and recent results}

By the Uniformization Theorem a genus $g$ with $n$ punctures, $2 g-2+n>0$, Riemann surface $R$ has a unique hyperbolic metric $d s^{2}$. By Riemann-Roch the associated space of holomorphic quadratic differentials $Q(R)$ on $R$ with at most simple poles at punctures has dimension $3 g-3+n$. By KodairaSpencer deformation theory and Serre duality $Q(R)$ is the dual of the space of infinitesimal deformations of $R$. Weil introduced a Hermitian pairing for 
the space of infinitesimal deformations by defining

$$
\langle\varphi, \psi\rangle=\int_{R} \varphi \bar{\psi}\left(d s^{2}\right)^{-1} \text { for } \varphi, \psi \in Q(R), \text { Ahl61]. }
$$

The Teichmüller space $\mathcal{T}$ is the space of homotopy-marked hyperbolic metrics for a surface of genus $g$ with $n$ punctures. The Weil-Petersson (WP) metric for $\mathcal{T}$ is invariant under the action of the mapping class group $M C G$ (the mapping class group is $\left.\mathrm{Homeo}^{+}(R) / \mathrm{Homeo}_{0}(R)\right)$. The metric is Kähler, non complete with negative sectional curvatures $\kappa$ with $\sup _{\mathcal{T}} \kappa=0$ (except for $\operatorname{dim}_{\mathbb{C}} \mathcal{T}=1$ ) and $\inf _{\mathcal{T}} \kappa=-\infty$. The metric completion of $\mathcal{T}$ is the augmented Teichmüller space $\overline{\mathcal{T}}$, the space of homotopy-marked noded Riemann surfaces. $\overline{\mathcal{T}}$ is a disjoint union of $\mathcal{T}$ and lower dimensional Teichmüller spaces. With the completion metric $\overline{\mathcal{T}}$ is an example of a $C A T(0)$ metric space, a simply connected generalized space of negative curvature, DW03, Wlp03. Alexandrov and others developed a collection of techniques for understanding $C A T(0)$ spaces, BH99. The topological pants graph $P(R)$ has a vertex for each collection of $3 g-3+n$ distinct free homotopy classes of non trivial, non peripheral, mutually disjoint simple closed curves. Vertices of $P(R)$ are connected by an edge provided the corresponding sets of free homotopy classes differ by replacing a single curve with a once or twice intersecting curve (a Hatcher-Thurston move). Brock discovered that Teichmüller space with the WP metric is quasi isometric to $P(R)$ with the unit-edge metric, Bro03. At large scale on Teichmüller space WP distance is combinatorially determined.

The importance of the Weil-Petersson metric begins with the natural family of Riemann surfaces over Teichmüller space being the universal family of homotopy-marked hyperbolic metrics. There are wide ranging applications of the geometry. The WP Kähler geometry provides a complex differential geometric proof with the Kodaira Embedding Theorem that the DeligneMumford compactification $\overline{\mathcal{T} / M C G}$ is projective algebraic, Wlp85a. The metric convexity provides for Nielsen Realization: finite subgroups of MCG acting on $\mathcal{T}$ have non empty fixed-point sets, Wlp87. Mirzakhani's WP volume recursion (based on symplectic reduction) in topological type $g, n$ provides for a solution of the Witten-Kontsevich conjecture, Mir07b, Mir07a. Li-Sun-Yau use the WP Ricci form as a beginning reference metric for constructing and estimating the Kähler-Einstein metric on $\mathcal{T}$, LSY04, LSY05]. They establish comparability of the Kähler-Einstein metric to the five classical complete metrics (Kobayashi-Teichmüller, Carathéodory, asymptotic Poincaré, McMullen and Bergman). Up to comparability the six metrics prospectively share properties. 
There are also wide ranging descriptions of the WP metric. For a deformation family of Riemann surfaces $R_{t}$ beginning with $R$, consider the energy $E\left(f_{t}\right)$ of the harmonic map $f_{t}: R \rightarrow R_{t}$. Up to a universal constant the Hessian of $E\left(f_{t}\right)$ is the WP metric at $R$, Wlf89. For the quasiFuchsian group $\Gamma_{t}$ representing the pair of Riemann surfaces $\left(R, R_{t}\right)$ then up to a constant the Hessian of the Hausdorff dimension of the limit set of $\Gamma_{t}$ is also the WP metric, McM06. There is also a characterization in terms of geodesic-length functions. For a geodesic $\gamma$ on $R$ let $\ell_{\gamma}\left(R_{t}\right)$ be the length of the unique hyperbolic metric geodesic in the corresponding free homotopy class on $R_{t}$. Let $\left\{\gamma_{j}\right\}$ be a sequence of geodesics with length-normalized lifts to the unit-tangent bundle $T R$ tending weak* to the uniform distribution. The $\ell_{\gamma_{j}}$ Hessians tend to the WP metric as follows (for $n=0$; Thurston's random geodesic metric is the WP metric)

$$
\langle,\rangle=3 \pi(g-1) \lim _{j} \frac{1}{\ell_{j}} \text { Hess } \ell_{\gamma_{j}} \text { Wlp86b. }
$$

\section{Weil-Petersson research themes}

The deformation theory of Riemann surfaces and hyperbolic metrics provides an entree to deformation theory of higher dimensional manifolds. Deformations of Riemann surfaces represent a basic case of the application of: Kodaira-Spencer theory; the prescribed curvature equation; geometric structures described by developing maps; $\operatorname{Hom}\left(\pi_{1}(R), G\right) / G(G=P S L(2 ; \mathbb{R})$ for hyperbolic metrics) and for Quillen's metric approach to the local index theorem for the complex differential (Cauchy-Riemann operator) $\bar{\partial}$.

A theme of investigation is intersection theory and understanding curvature. Intersection theory of cycles on the Deligne-Mumford compactification $\overline{\mathcal{T} / M C G}$ was employed, Wlp83a, to evaluate the WP class and is important to Penner's resolution of Witten's conjecture on an explicit cycle Poincaré dual to the WP Kähler form, Pen93. The sectional curvature was originally considered by Royden, Tromba and the author, Roy75, Tro86, Wlp86a. Recent results include that the WP metric is Gromov hyperbolic iff $\operatorname{dim}_{\mathbb{C}} \mathcal{T} \leq 2$, BF06]. Zheng Huang has been effecting a detailed analysis of WP curvature, Hua05, Hua07a, Hua07b, GGHar. While the WP volume grows exponentially in $g$, he finds that on the thick part of the moduli spaces the curvatures are bounded. A precise application of curvature and the hyperbolic metric is given Freixas' development of arithmetic intersection theory following Gillet-Soulé and Bismut for Riemann surfaces with punctures, FiM09. A 
consequence is the exact formula for the Selberg zeta function

$$
Z^{\prime}(1)=4 \pi^{5 / 3} \Gamma_{2}(1 / 2)^{-8 / 3}
$$

for the principal subgroup $\Gamma(2) \subset S L(2 ; \mathbb{Z})$ and the Barnes double gamma function. The works of Takhtajan and Zograf present an original application of Quillen's metric for calculating the Chern form of the determinant line bundle, [TZ87, TZ91, TZ88]. The determinant is given by a special value of the Selberg zeta function.

An important theme of investigation is the WP symplectic geometry. An original result is the duality $\omega\left(t_{\gamma}, \quad\right)=-\frac{1}{2} d \ell_{\gamma}$ of Fenchel-Nielsen infinitesimal deformations and geodesic-length functions relative to the WP symplectic form $\omega$, Wlp83b. The symplectic geometry is manifested in the formula for Fenchel-Nielsen twist-length coordinates

$$
\omega=\frac{1}{2} \sum_{\gamma_{j}} d \ell_{j} \wedge d \tau_{j}, \text { Wlp85b, }
$$

where $\left\{\gamma_{j}\right\}$ gives a pants decomposition (the expression is universal even though there are an infinite number of topologically distinct pants decompositions). Goldman more generally considered trace-functions on the representation spaces $\operatorname{Hom}\left(\pi_{1}(R), G\right) / G$ as Hamiltonians relative to the cup product for the group-cohomology description of the tangent space, Gol86].

In a series of innovative papers Mirzakhani presented calculations of WP integrals exploiting the underlying symplectic geometry, Mir07a, Mir08, Mir07b. She also established a prime geodesic theorem for simple geodesics $\#\left\{\gamma \mid \ell_{\gamma}(R) \leq L\right\} \sim c_{R} L^{6 g-6+2 n}$, for $L$ large, and a proof of the Witten Kontsevich formulas for intersections of tautological classes on the DeligneMumford compactification $\overline{\mathcal{T} / M C G}$. Her work is an entree to several matters. She combines a generalization of McShane's universal length-sum identity, recursions of integrals, Thurston's symplectic geometry for the space of measured geodesic laminations and symplectic reduction of quasi-free $S^{1}$ actions to obtain a collection of new results. She finds volume formulas for the moduli spaces of hyperbolic surfaces with geodesic boundary including the formula

$$
\operatorname{vol}_{W P}=\frac{\pi^{2}}{6}+\frac{b^{2}}{24}
$$

for the moduli space of tori with length $b$ boundary.

Another theme of WP investigation is the metric space geometry and the behavior of geodesics. As above the augmented Teichmüller space $\overline{\mathcal{T}}$ is a $C A T(0)$ metric space with $\mathcal{T}, \overline{\mathcal{T}}$ quasi isometric to the pants graph $P(R)$. 
Recently the Alexandrov tangent cones of $\overline{\mathcal{T}}$ have been described, Wlp08. The description provides for applications and for considering combinatorial harmonic maps into $\overline{\mathcal{T}}$. A refined study of WP geodesics is now underway. Brock-Masur-Minsky have introduced a notion of ending lamination for infinite WP rays and apply their notion to embed asymptote classes (of recurrence rays on $\mathcal{T} / M C G$ ) into the Gromov boundary of the curve complex $C(R)$ (the complex with $k$ simplices being collections of $k+1$ distinct free homotopy classes of non trivial, non peripheral, mutually disjoint simple closed curves on $R$ ), BMM07. The authors apply their analysis to show that the WP geodesic flow is topologically transitive. Pollicott-WeissWolpert use a form of the classical closing lemma for geodesics on surfaces to obtain a geometric elementary proof for $\operatorname{dim}_{\mathbb{C}} \mathcal{T}=1$, [PWW10].

\section{Weil-Petersson research techniques}

A range of techniques is applied for the study of variations of hyperbolic metrics and WP geometry. A majority of approaches have a basis in complex analysis and complex differential geometry. Deformation theory plays the largest role.

An application of the Dolbeault complex is that the potential equation $\bar{\partial} f=\mu$ is central to deformation theory for surfaces. The Uniformization Theorem provides solutions for the prescribed curvature equation. The variation theory for the prescribed curvature equation provides an approach for deformations. The Eells-Sampson variation theory for harmonic maps provides a closely related approach. Riemann surfaces are also described as identification spaces. Deformations of geometric structures are correspondingly described as Čech 1-cocycles with values in special vector fields. The Čech plates viewpoint provides a context for describing the Fenchel-Nielsen twist deformation, as well as Thurston's earthquake and Penner's shearing. There is also a description of deformations following the classical theory of automorphic forms; the approach is based on explicit evaluations of integrals and sums - and expressing results in geometric terms, Wlp83b. A basic consideration is a sum over configuration of lines in the hyperbolic plane.

Many WP results are based on considerations of (complex) differential geometry - for the individual Riemann surfaces - and for Teichmüller space. Li-Sun-Yau employ complex differential geometry, analysis and comparison

arguments including the Schwarz Lemma of Yau. Recently techniques of synthetic geometry and especially $C A T(0)$ geometry have also become important, Bro05, BF06, BM07, BMM07, DW03, Wlp03, Wlp08. The inter- 
section theory considerations involve differential forms, symplectic geometry and extensive considerations of Greens functions. In fact Greens functions appear in the expressions for WP curvature, curvature of the family hyperbolic metric Whlp86a, formulas of bosonic Polyakov string theory [DP86], and in the form of Eisenstein series in the Takhtajan-Zograf local index formula [TZ91, and in the curvature of the renormalized family hyperbolic metric Wlp07. Understanding Greens functions is basic for understanding curvature.

Topological considerations are also used to investigate WP geometry. The pants graphs $P(R)$, curve complex $C(R)$ and measured geodesic laminations play important roles. Mirzakhani's considerations involve Thurston's symplectic form on the space of measured geodesic laminations. BrockMasur-Minsky employ methods developed in examination of Thurston's ending lamination conjecture.

Dynamical considerations are also used. McMullen's approach reveals a connection between Hausdorff dimension, norms of holomorphic forms, and the central limit theorem for geodesic flows, especially the variance of observables of mean zero, McM06. The Brock-Minsky-Masur considerations on limits of sequences in $C(R)$ include beginning dynamical arguments.

\section{$5 \quad$ Lecture material}

- Basics: hyperbolic surfaces, deformations and topological methods (2 lectures)

- Teichmüller space, models for infinitesimal deformations; WeilPetersson and Teichmüller metrics; mapping class groups; moduli space; Deligne-Mumford compactification

- constructing surfaces and hyperbolic metrics; enhanced collar lemma and simple geodesics; Fenchel-Nielsen deformation; geodesiclength functions; twist-length duality; first and second twist derivatives of geodesic-length functions

- measured geodesic laminations; Thurston's symplectic form; curve complex and pants graph; Gromov boundary of the curve complex; ending lamination methods

- Estimating coset sums of inverse-exponential-distance (including estimating Greens functions) 
- examples of inverse-exponential sums; enhanced mean value estimate; estimating on thick and thin regions

- Geodesic-length functions (1 1/2 lectures)

- cosine formula; Riera's formula; Hessian of geodesic-length; convexity; small-length expansions; special Schwarz lemma; expansion of WP Levi Civita connection; applications

- Augmented Teichmüller space $\overline{\mathcal{T}}$ and $C A T(0)$ (1 1/2 lectures)

- definition $\overline{\mathcal{T}} ; C A T(0)$; Brock's approximation of geodesics; Brock's quasi isometry; $\overline{\mathcal{T}}$ as an infinite polyhedra; $M C G=\operatorname{Isom}_{W P}$; classification of flats; Alexandrov tangent cones

- Mirzakhani's volume recursion and prime geodesic theorem (1 1/2 lectures)

- Mirzakhani-McShane identity; the recursion of volume integrals; simple geodesics and counting lattice points on MGL; MakoverMcGowan's expected short length statistics

- Behavior of WP geodesics and topological transitivity of geodesic flow

- compactness theorem for geodesics; ending laminations for infinite WP geodesics; recurrent WP geodesics; encoding geodesics

- Curvature and index formulas for families hyperbolic metrics ( $11 / 2$ lectures)

- formulas; perturbations of the prescribed curvature equation; degenerating curvatures; the plumbing family $\{z w=t\} \rightarrow\{t\}$; families of holomorphic 2-differentials; Mumford's good \& pre$\log -\log$ metrics 


\section{References}

[Ahl61] Lars V. Ahlfors. Some remarks on Teichmüller's space of Riemann surfaces. Ann. of Math. (2), 74:171-191, 1961.

[BF06] Jeffrey Brock and Benson Farb. Curvature and rank of Teichmüller space. Amer. J. Math., 128(1):1-22, 2006.

[BH99] Martin R. Bridson and André Haefliger. Metric spaces of nonpositive curvature. Springer-Verlag, Berlin, 1999.

[BM07] Jeffrey Brock and Howard Masur. Coarse and synthetic WeilPetersson geometry: quasi-flats, geodesics, and relative hyperbolicity. preprint, 2007.

[BMM07] Jeffrey Brock, Howard Masur, and Yair Minsky. Asymptotics of Weil-Petersson geodesics I: ending laminations, recurrence, and flows. preprint, 2007.

[Bro03] Jeffrey F. Brock. The Weil-Petersson metric and volumes of 3-dimensional hyperbolic convex cores. J. Amer. Math. Soc., 16(3):495-535 (electronic), 2003.

[Bro05] Jeffrey F. Brock. The Weil-Petersson visual sphere. Geom. Dedicata, 115:1-18, 2005.

[DP86] Eric D'Hoker and D. H. Phong. Multiloop amplitudes for the bosonic Polyakov string. Nuclear Phys. B, 269(1):205-234, 1986.

[DW03] Georgios Daskalopoulos and Richard Wentworth. Classification of Weil-Petersson isometries. Amer. J. Math., 125(4):941-975, 2003.

[FiM09] Gérard Freixas i Montplet. An arithmetic Riemann-Roch theorem for pointed stable curves. Ann. Sci. Éc. Norm. Supér. (4), 42(2):335-369, 2009.

[GGHar] Ren Guo, Subhojoy Gupta, and Zheng Huang. Curvatures on the Teichmüller curve. Indiana Univ. Math. Jour., to appear.

[Gol86] William M. Goldman. Invariant functions on Lie groups and Hamiltonian flows of surface group representations. Invent. Math., 85(2):263-302, 1986. 
[Hua05] Zheng Huang. Asymptotic flatness of the Weil-Petersson metric on Teichmüller space. Geom. Dedicata, 110:81-102, 2005.

[Hua07a] Zheng Huang. On asymptotic Weil-Petersson geometry of Teichmüller space of Riemann surfaces. Asian J. Math., 11(3):459484, 2007.

[Hua07b] Zheng Huang. The Weil-Petersson geometry on the thick part of the moduli space of Riemann surfaces. Proc. Amer. Math. Soc., 135(10):3309-3316 (electronic), 2007.

[LSY04] Kefeng Liu, Xiaofeng Sun, and Shing-Tung Yau. Canonical metrics on the moduli space of Riemann surfaces. I. J. Differential Geom., 68(3):571-637, 2004.

[LSY05] Kefeng Liu, Xiaofeng Sun, and Shing-Tung Yau. Canonical metrics on the moduli space of Riemann surfaces. II. J. Differential Geom., 69(1):163-216, 2005.

[McM06] Curtis T. McMullen. Thermodynamics, dimension and the WeilPetersson metric. preprint, 2006.

[Mir07a] Maryam Mirzakhani. Simple geodesics and Weil-Petersson volumes of moduli spaces of bordered Riemann surfaces. Invent. Math., 167(1):179-222, 2007.

[Mir07b] Maryam Mirzakhani. Weil-Petersson volumes and intersection theory on the moduli space of curves. J. Amer. Math. Soc., 20(1):1-23 (electronic), 2007.

[Mir08] Maryam Mirzakhani. Growth of the number of simple closed geodesics on hyperbolic surfaces. Ann. of Math. (2), 168(1):97$125,2008$.

[MM05] Eran Makover and Jeffrey McGowan. The length of closed geodesics on random Riemann surfaces. Arxiv:math/0504175, 2005.

[Pen93] Robert C. Penner. The Poincaré dual of the Weil-Petersson Kähler two-form. Comm. Anal. Geom., 1(1):43-69, 1993.

[PWW10] Mark Pollicott, Howard Weiss, and Scott A. Wolpert. Topological dynamics of the Weil-Petersson geodesic flow. Adv. Math., 223(4):1225-1235, 2010. 
[Roy75] H. L. Royden. Intrinsic metrics on Teichmüller space. In Proceedings of the International Congress of Mathematicians (Vancouver, B. C., 1974), Vol. 2, pages 217-221. Canad. Math. Congress, Montreal, Que., 1975.

[Tro86] A. J. Tromba. On a natural algebraic affine connection on the space of almost complex structures and the curvature of Teichmüller space with respect to its Weil-Petersson metric. Manuscripta Math., 56(4):475-497, 1986.

[TZ87] L. A. Takhtadzhyan and P. G. Zograf. A local index theorem for families of $\bar{\partial}$-operators on Riemann surfaces. Uspekhi Mat. Nauk, 42(6(258)):133-150, 248, 1987.

[TZ88] L. A. Takhtajan and P. G. Zograf. The Selberg zeta function and a new Kähler metric on the moduli space of punctured Riemann surfaces. J. Geom. Phys., 5(4):551-570 (1989), 1988.

[TZ91] L. A. Takhtajan and P. G. Zograf. A local index theorem for families of $\bar{\partial}$-operators on punctured Riemann surfaces and a new Kähler metric on their moduli spaces. Comm. Math. Phys., 137(2):399-426, 1991.

[Wlf89] Michael Wolf. The Teichmüller theory of harmonic maps. $J$. Differential Geom., 29(2):449-479, 1989.

[Wlp83a] Scott A. Wolpert. On the homology of the moduli space of stable curves. Ann. of Math. (2), 118(3):491-523, 1983.

[Wlp83b] Scott A. Wolpert. On the symplectic geometry of deformations of a hyperbolic surface. Ann. of Math. (2), 117(2):207-234, 1983.

[Wlp85a] Scott A. Wolpert. On obtaining a positive line bundle from the Weil-Petersson class. Amer. J. Math., 107(6):1485-1507 (1986), 1985.

[Wlp85b] Scott A. Wolpert. On the Weil-Petersson geometry of the moduli space of curves. Amer. J. Math., 107(4):969-997, 1985.

[Wlp86a] Scott A. Wolpert. Chern forms and the Riemann tensor for the moduli space of curves. Invent. Math., 85(1):119-145, 1986.

[Wlp86b] Scott A. Wolpert. Thurston's Riemannian metric for Teichmüller space. J. Differential Geom., 23(2):143-174, 1986. 
[Wlp87] Scott A. Wolpert. Geodesic length functions and the Nielsen problem. J. Differential Geom., 25(2):275-296, 1987.

[Wlp03] Scott A. Wolpert. Geometry of the Weil-Petersson completion of Teichmüller space. In Surveys in Differential Geometry VIII: Papers in Honor of Calabi, Lawson, Siu and Uhlenbeck, pages 357-393. Intl. Press, Cambridge, MA, 2003.

[Wlp07] Scott A. Wolpert. Cusps and the family hyperbolic metric. Duke Math. J., 138(3):423-443, 2007.

[Wlp08] Scott A. Wolpert. Behavior of geodesic-length functions on Teichmüller space. J. Differential Geom., 79(2):277-334, 2008.

[Wlp09] Scott A. Wolpert. Extension of the Weil-Petersson connection. Duke Math. J., 146(2):281-303, 2009. 